\title{
PRODUÇÃO E COLABORAÇÃO CIENTÍFICA DA UNIVERSIDADE FEDERAL DE CIÊNCIAS DA SAÚDE DE PORTO ALEGRE
}

\author{
PRODUCCIÓN Y COLABORACIÓN CIENTÍFICA DE LA \\ UNIVERSIDADE FEDERAL DE CIÊNCIAS DA SAÚDE DE \\ PORTO ALEGRE
}

\author{
Maiara Bettio* \\ Gonzalo Rubén Alvarez** \\ Samile Andréa de Souza Vanz ${ }^{* * *}$
}

\begin{abstract}
RESUMO
Introdução: O mapeamento da produção científica das instituições de ensino e pesquisa é fundamental para planejamento de políticas e avaliação de desempenho. Objetivo: Identificar e caracterizar a produção científica da Universidade Federal de Ciências da Saúde de Porto Alegre (UFCSPA) através deindicadores bibliométricos de produção e de colaboração. Metodologia: Análise bibliométrica de 1.932 documentos indexados na base de dados Web of Science (WoS) de 1961 a 2014, com auxílio dos softwares Bibexcel e Excel 2010. Resultados: A análise revela o crescimento anual significativo das publicaçõesda instituição (33,5\% ao ano), destacando-se o período 2008-2014. O artigo foi utilizado em $70,9 \%$ das publicações e $91,3 \%$ dos documentos foram publicados no idioma inglês. As áreas de conhecimento com maior produtividade foram a Cirurgia e as Neurociências. A autoria múltipla mostra-se predominante $(98,6 \%)$. Destes, $17,7 \%$ apresentam-se em coautoria internacional. Estados Unidos, Reino Unido, Canadá, Espanha, França e Argentina são os principais parceiros. Dos 1.905 documentos com colaboração, 86,8\% contam com duas ou maisinstituições coautoras, com predomínio das universidades nacionais públicas, além das instituições hospitalares. UFRGS $(41,6 \%)$ e Santa Casa de Misericórdiade Porto Alegre $(31,0 \%)$ se destacam como as principais parceiras. Conclusões: Conclui que o aumento dos grupos de pesquisa e docentes pesquisadores contribuíram para 0 crescimento da produção intelectual da UFCSPA.
\end{abstract}

Palavras-chave: Bibliometria. Produtividade científica. Colaboração científica. Universidade Federal de Ciências da Saúde de Porto Alegre. Medicina.

*Mestranda do programa de pós-graduação em Comunicação e informação da Universidade Federal do Rio Grande do Sul. E-mail: maiarabettio@yahoo.com.br

**Doutorando do programa de pós-graduação em Comunicação e informação da Universidade Federal do Rio Grande do Sul. E-mail: gonzalorubenalvarez@gmail.com

***Docente do Programa de Pós Graduação em Comunicação e Informação da Universidade Federal do Rio Grande do Sul. E-mail: samilevanz@terra.com.br 


\section{INTRODUÇÃO}

Desde seu surgimento no século $\mathrm{XI}$, as universidades são distinguidas como ambientes de transmissão do saber. Elas evoluíram tendo como uma de suas funções básicas a produção de conhecimento por meio da investigação científica da qual participam professores/pesquisadores e alunos (STUMPF, 2000), gerando pesquisas que resultam no desenvolvimento de produtos e tecnologias capazes de melhorar as condições sociais e econômicas da população (MEIS; LETA, 1996).

Schwartzman (2001) expressa que apenas no século XIX a ciência adquiriu a forma que conhecemos hoje, fortemente atrelada à pesquisa e ao ambiente acadêmico. Foi na Alemanha que a pesquisa científica foi introduzida ao ambiente universitário, dando origem a um sistema educacional integrado, que serviria de modelo para outros países. No século $X X$, teve início o processo de institucionalização da pesquisa no Brasil e ocorreu a fundação da Universidade de São Paulo (USP), em 1934. Com o objetivo de promover o progresso da ciência através da pesquisa, a criação da USP foi um marco na história da ciência e da educação no país (MEIS; LETA, 1996).

Componente essencial da atividade científica, a avaliação da produção gerada na universidade visaa garantir o investimento financeiro em pesquisa e a participação da ciência na consecução dos objetivos econômicos, sociais e políticos do país (VELHO, 1986). A avaliação pode ser realizadaatravés de análises métricas, utilizando indicadores fornecidos pela Cientometria e Bibliometria, áreas que estudam os aspectos quantitativos da ciência enquanto atividade econômica e da produção, disseminação e uso da informação registrada (MACIAS-CHAPULA, 1998).

A Universidade Federal de Ciências da Saúde de Porto Alegre é uma Instituição Federal de Ensino Superior especializada na área da saúde (UFCSPA, 2015b) e está localizada na área central de Porto Alegre, capital do estado do Rio Grande do Sul. Criada em 1953 como Faculdade Católica de Medicina de Porto Alegre e em atividade desde 1961, a faculdade tinha como objetivo inicial a formação de novos médicos para atender à crescente demanda por estes profissionais dentro da instituição mantenedora da época, a 
Maiara Bettio, Gonzalo Rubén Alvarez, Samile Andréa de Souza Vanz

Produção e colaboração científica da Universidade Federal de Ciências da Saúde de

Porto Alegre

Santa Casa de Misericórdia de Porto Alegre (Santa Casa) (UFCSPA, 2015a). A faculdade foi federalizada em 1980, denominada Fundação Faculdade Federal de Ciências Médicas de Porto Alegre (FFFCMPA). A Santa Casa deixou de ser sua mantenedora, mas manteve vínculo através das atividades de ensino desenvolvidas em seu complexo hospitalar, em nível técnico, de graduação, especialização, mestrado e doutorado (BONAMIGO; OLIVEIRA, 2007).

Em 2008 a instituição tornou-se universidade e passou a se chamar Universidade Federal de Ciências da Saúde de Porto Alegre (UFCSPA, 2015a). Com qualidade nacional reconhecida, a UFCSPA visa ao cumprimento da missão de ensinar, pesquisar e estender serviços à sociedade, formando profissionais de variadas especialidades em Ciências da Saúde (UFCSPA, 2014). De acordo com o Exame Nacional de Desempenho dos Estudantes (Enade) e o Índice Geral de Cursos (IGC) do Ministério da Educação, no ano 2013 a UFCSPA encontrava-se entre as melhores universidades federais do Rio Grande do Sul e foi considerada a terceira melhor universidade do país na categoria graduação (UFCSPA, 2015c). Em 2014, sua comunidade acadêmica era formada por 355 professores, 196 técnicos administrativos e 3.674 alunos matriculados em cursos de graduação e de pós-graduação (UFCSPA, 2015e), números que vêm crescendo a cada ano devido à política de desenvolvimento institucional que tem ampliado a oferta de cursos na universidade.

Na modalidade de graduação, o histórico de expansão da UFCSPA é recente, visto que apenas em 2004 deu-se a criação de novos cursos, indo além do tradicional curso de Medicina criado em 1961. Desde sua transformação em universidade, esse fenômeno tem se acelerado, com a criação de outros cursos de graduação. Atualmente são 14 cursos de graduação oferecidos pela instituição.

$\mathrm{Na}$ pós-graduação, a universidade oferece cursos nas modalidades profissional (residência médica e residência multiprofissional), Lato sensu (especialização) e Strictu sensu (mestrado e doutorado). A oferta de cursos de pós-graduação Stricto sensu teve início em 1988, com a criação do curso de Pós-Graduação em Farmacologia, em nível de mestrado. Posteriormente, em 1993, os primeiros cursos de doutorado passaram a ser oferecidos nas áreas de Farmacologia e de Patologia (BONAMIGO; OLIVEIRA, 2007). Atualmente, a 
UFCSPA oferece noveprogramas em nível de mestrado e cinco em nível de doutorado.

Além disso, entre 2000 e 2014, a UFCSPA triplicou o número de grupos de pesquisa vinculados ao Diretório de Grupos de Pesquisa do CNPq. Atualmente, a instituição conta com 39 grupos, representados nas seguintes áreas: Ciências da Saúde, Ciências Biológicas, Ciências Humanas, Ciências Agrárias, Linguística, Letras e Artes e Sociais Aplicadas (CNPq, 2015).

A instituição demonstra ainda uma forte preocupação com o crescimento e a qualificação de sua pesquisa, evidente na análise das metas apresentadas no Plano de Metas das Unidades (UFCSPA, 2015d) e no Plano de Desenvolvimento Institucional (UFCSPA, 2015d). Entre as ações propostas, destaca-se o incentivo e a ampliação da produção científica, bem como sua avaliação periódica e a divulgação dos indicadores de desempenho e de produção. De acordo com o Relatório de Gestão publicado em 2015, os pesquisadores da UFCSPA vêm sendo fortemente instados a publicar, de preferência, em periódicos internacionais. Existe, por parte da gestão atual, a expectativa de que a produção científica indexada em bases internacionais aumente nos próximos anos, devido à consolidação das carreiras dos novos pesquisadores, à melhoria da infraestrutura institucional de pesquisa, assim como ao aporte de recursos de fomento federais e estaduais.

Nesse sentido, este estudo busca analisar as características daprodução científica da UFCSPA através de indicadores de atividade e colaboração no período de 1961 a 2014, buscando promover sua visibilidade e evidenciando a importância do trabalho acadêmico desenvolvido pelos pesquisadores da universidade. As seções a seguir apresentam a metodologia, os resultados e as conclusões dessa análise.

\section{METODOLOGIA}

O corpus de análise deste estudo é composto pelas publicações da UFCSPA indexadas na base de dados multidisciplinar Web of Science (WoS) no período de 1961-2014. A busca e coleta desses registros foram realizadas em 27 de agosto de 2015, através da opção de Busca Avançada, nos índices Science Citation Index Expanded, Social Sciences Citation Index, Arts \& 
Maiara Bettio, Gonzalo Rubén Alvarez, Samile Andréa de Souza Vanz

Produção e colaboração científica da Universidade Federal de Ciências da Saúde de

Porto Alegre

Humanities Citation Index, Conference Proceedings Citation Index e Conference Proceedings Citation Index - Social Science \& Humanities, incluindo todos os tipos de documentos e idiomas. Delimitou-se o período de 1961 - ano de início das atividades da UFCSPA - até 2014, no entanto, os primeiros registros contendo a filiação de autores à universidade apareceram em 1979.

A estratégia de busca incorporou variantes tanto do nome atual da universidade quanto dos antigos nomes a ela atribuídos ao longo de sua história. Devido ao forte vínculo de colaboração da UFCSPA com a Santa Casa, também foi realizada a busca por variantes do nome do complexo hospitalar, com análise individual destes registros para averiguar possíveis autores que tenham omitido sua relação com a universidade. A Plataforma Lattes do CNPq e a lista de docentes da universidade (corrente e retrospectiva) foram consultadas para a confirmação do vínculo institucional de autores de publicações da Santa Casa, bem como de publicações com variantes muito genéricas do nome da universidade. A Lista de Autoridades do Grupo de Pesquisa Comunicação Científica da Universidade Federal do Rio Grande do Sul (UFRGS) foi utilizada para a construção da estratégia de busca e para a padronização do nome das instituições colaboradoras.

Foram identificados 1.932 registros bibliográficos, analisados a partir de indicadores bibliométricos de produção (ano de publicação, tipologia documentária, idioma, periódico, área de conhecimento eprodutividade de autores) e de colaboração (coautoria entre autores, instituições e países). Para tratamento dos dados foram utilizados os softwares Bibexcel e Microsoft Excel 2010. O Journal Citation Reports foi consultado para levantamento dos dados e indicadores referentes aos periódicos.

\section{RESULTADOS}

A análise da produção científica da UFCSPA representada na Web of Sciencerevela 1.932 publicações, apresentadas a seguir a partir de indicadores de produção e colaboração. 
Maiara Bettio, Gonzalo Rubén Alvarez, Samile Andréa de Souza Vanz

Produção e colaboração científica da Universidade Federal de Ciências da Saúde de Porto Alegre

\subsection{Indicadores de produção}

A Tabela 1 apresenta o panorama do número de documentos por ano e as respectivas porcentagensde crescimento em relação ao ano anterior, o número de grupos de pesquisa, programas de mestrado e doutorado e cursos de graduação da UFCSPA ao longo dos anos.

Tabela 1 - Número de documentos da UFCSPA indexados na WoS, grupos de pesquisa, programas de mestrado ou doutorado, docentes na pós-graduação e cursos de graduação (1961-2014)

\begin{tabular}{|c|c|c|c|c|c|c|}
\hline $\begin{array}{c}\text { Ano de } \\
\text { publicação }\end{array}$ & $\begin{array}{c}\text { N. de } \\
\text { documentos } \\
\text { na WoS }\end{array}$ & $\begin{array}{l}\% \text { de } \\
\text { crescimento em } \\
\text { relação ao ano } \\
\text { anterior }\end{array}$ & $\begin{array}{l}\text { N. de grupos } \\
\text { de pesquisa* }\end{array}$ & $\begin{array}{c}\text { N. de } \\
\text { programas } \\
\text { de mestrado } \\
\text { elou } \\
\text { doutorado } \\
\end{array}$ & $\begin{array}{l}\text { N. de docentes } \\
\text { na pós- } \\
\text { graduação* }\end{array}$ & $\begin{array}{c}\text { N. de } \\
\text { cursos de } \\
\text { graduaçãao }\end{array}$ \\
\hline 1961 a 1978 & 0 & - & - & 0 & - & 1 \\
\hline 1979 & 1 & - & - & 0 & - & 0 \\
\hline 1980 & 0 & $-100,0$ & - & 0 & - & 0 \\
\hline 1981 & 0 & 0,0 & - & 0 & - & 0 \\
\hline 1982 & 5 & 500,0 & - & 0 & - & 0 \\
\hline 1983 & 2 & $-60,0$ & - & 0 & - & 0 \\
\hline 1984 & 3 & 50,0 & - & 0 & - & 0 \\
\hline 1985 & 5 & 66,7 & - & 0 & - & 0 \\
\hline 1986 & 4 & $-20,0$ & - & 0 & - & 0 \\
\hline 1987 & 1 & $-75,0$ & - & 0 & - & 0 \\
\hline 1988 & 3 & 200,0 & - & 1 & - & 0 \\
\hline 1989 & 3 & 0,0 & - & 0 & - & 0 \\
\hline 1990 & 4 & 33,3 & - & 0 & - & 0 \\
\hline 1991 & 5 & 25,0 & - & 0 & - & 0 \\
\hline 1992 & 11 & 120,0 & - & 0 & - & 0 \\
\hline 1993 & 8 & $-27,3$ & - & 4 & - & 0 \\
\hline 1994 & 7 & $-12,5$ & - & 0 & - & 0 \\
\hline 1995 & 14 & 100,0 & - & 0 & - & 0 \\
\hline 1996 & 18 & 28,6 & - & 0 & - & 0 \\
\hline 1997 & 17 & $-5,6$ & - & 0 & - & 0 \\
\hline 1998 & 26 & 52,9 & - & 0 & - & 0 \\
\hline 1999 & 38 & 46,2 & - & 0 & - & 0 \\
\hline 2000 & 29 & $-23,7$ & 13 & 0 & - & 0 \\
\hline 2001 & 30 & 3,4 & - & 0 & - & 0 \\
\hline 2002 & 38 & 26,7 & 15 & 0 & - & 0 \\
\hline 2003 & 32 & $-15,8$ & - & 0 & - & 0 \\
\hline 2004 & 62 & 93,8 & 19 & 0 & - & 2 \\
\hline 2005 & 46 & $-25,8$ & - & 1 & - & 0 \\
\hline
\end{tabular}

Inf. Inf., Londrina, v. 22, n. 1, p. 88 - 110, jan./abr., 2017. 
Maiara Bettio, Gonzalo Rubén Alvarez, Samile Andréa de Souza Vanz

Produção e colaboração científica da Universidade Federal de Ciências da Saúde de Porto Alegre

\begin{tabular}{cccccccc}
\hline 2006 & 82 & 78,3 & 21 & 0 & - & 0 \\
\hline 2007 & 134 & 63,4 & - & 0 & - & 1 \\
\hline 2008 & 129 & $-3,7$ & 28 & 0 & 52 & 0 \\
\hline 2009 & 137 & 6,2 & - & 0 & 56 & 3 \\
2010 & 194 & 41,6 & 35 & 0 & 55 & 1 \\
\hline 2011 & 168 & $-13,4$ & - & 1 & 59 & 0 \\
\hline 2012 & 188 & 11,9 & - & 0 & 67 & 2 \\
\hline 2013 & 219 & 16,5 & - & 0 & 76 & 0 \\
\hline 2014 & 269 & 22,8 & 39 & 0 & 97 & 4 \\
\hline Total & 1.932 & - & 39 & 7 & 97 & 14 \\
\hline
\end{tabular}

Fonte: dados da pesquisa

* Números acumulados

Apesar da intenção de caracterizar a produção científica desde 1961, ano de criação da UFCSPA, até 1979 não foram identificadas publicações indexadas. Isso indica um desenvolvimento tardio das atividades de pesquisa na instituição, vistoque já haviam sido criados órgãos e agências de financiamento à pesquisa na década de 1950. Essa evolução pode estar relacionada ao histórico institucional da UFCSPA, cuja criação tinha como objetivo principal a formação de novos médicos para atuar na Santa Casa. A oferta de cursos de pós-graduação Stricto sensuteve início apenas em 1988, com a criação do curso de Pós-Graduação em Farmacologia, em nível de mestrado.

No Gráfico 1, verifica-se a evolução contínua do número de publicações da UFCSPA entre 1979 e 2014. Apesar da inexistência de publicações no biênio 1980-1981 e do número de ocorrências com taxas negativas, não houve comprometimento no crescimento para todo o período analisado, que alcançou um total de $268,0 \%$, com taxa média de $33,5 \%$ ao ano. Ao se analisar a taxa de crescimento médio de 10,7\% apontado por Almeida e Guimarães (2013) para a produção científica brasileira entre 1980 e 2010, pode-se avaliar positivamente os resultados alcançados pelas publicações da UFCSPA, cujo índice é três vezes maior. 
Maiara Bettio, Gonzalo Rubén Alvarez, Samile Andréa de Souza Vanz

Produção e colaboração científica da Universidade Federal de Ciências da Saúde de Porto Alegre

Gráfico 1 - Evolução da produção científica da UFCSPA indexada na WoS (19792014)

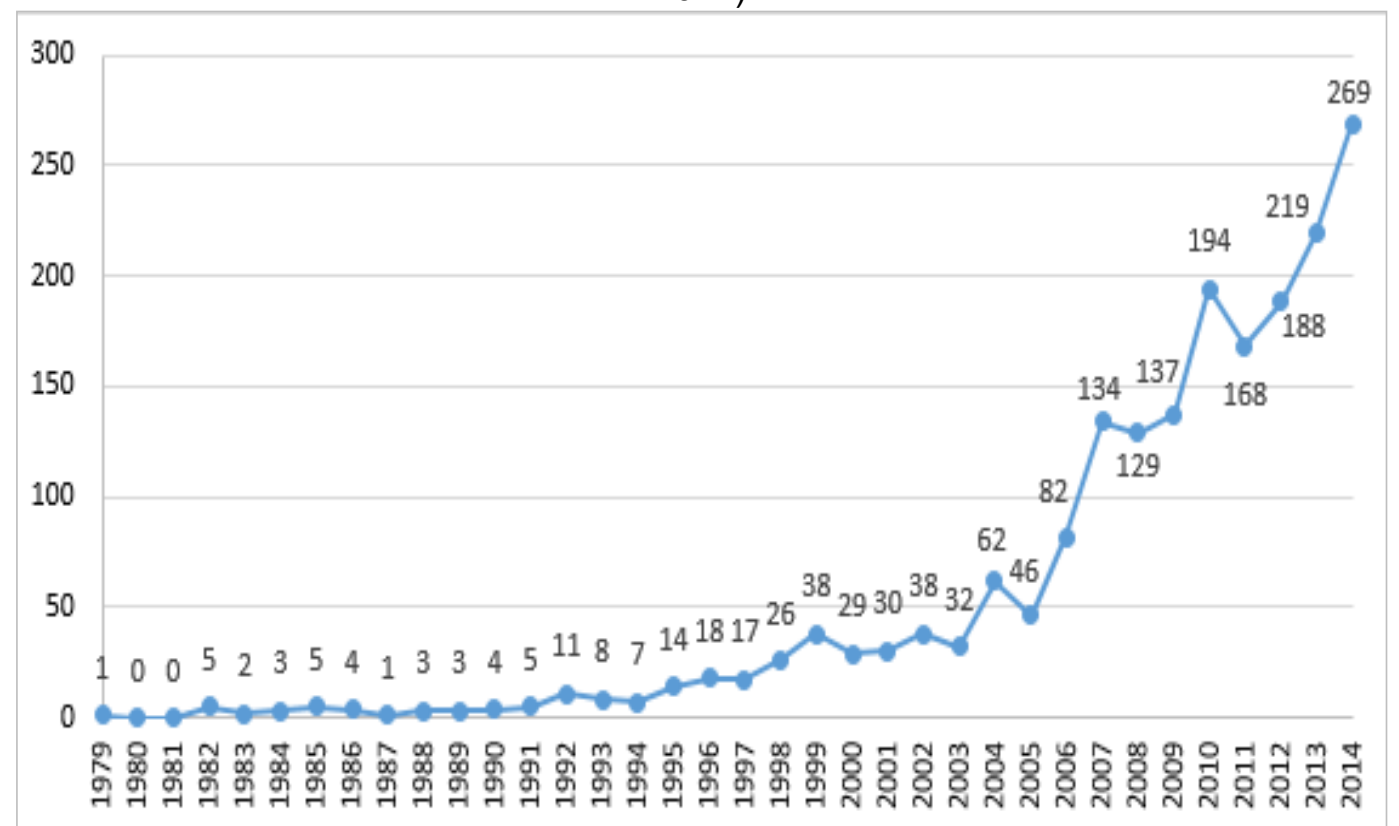

Fonte: dados da pesquisa

Entre 1979 e 1991, houve uma variação pouco expressiva de publicações. Umaumento significativo ocorre na década de 1990, que acumulou um crescimento total de $850,0 \%$, possivelmente como resultado da implementação do primeiro programa de pós-graduação da instituição, em 1988, e de outros dois novos programas em 1993, com cursos em nível de mestrado e doutorado nas áreas de Farmacologia, Patologia e Hepatologia. A correlação entre o crescimento da pós-graduação no país e o rápido crescimento da produção científica brasileira na WoS a partir do final da década de 1980 já foi demonstrada em outros estudos (MEIS; LETA, 1996).

A análise da produção publicada entre os anos 2000 e 2009 revela um crescimento expressivo e constante no número de documentos na WoS, com taxa média de 20,3\%. Tal desempenho é superior ao da UFRGS, universidade vizinha à UFCSPA e que apresentou crescimento médio de $12,69 \%$ no mesmo período (BRAMBILLA, 2011).

Ao se comparar os dados de crescimento observados pela FAPESP (2011) para a produção científica brasileira na WoS entre os anos 2002 e 2006, que corresponde a $43,5 \%$, verifica-se que a produção científica da UFCSPA no 
mesmo período obteve um crescimento expressivamente superior, de 115,7\%. Em relação à produção científica brasileira nas Ciências da Saúde, também indexada na base de dados do ISI, Saes (2005) verificou que houve um crescimento de 422,0\% no número de publicações da área entre 1990 e 2002. Comparada ao mesmo período, a produção científica da UFCSPA cresceu mais que o dobro, $850,0 \%$.

O período entre 2008 e 2014 merece destaque por concentrar $67,5 \%$ de todas as publicações da UFCSPA na WoS. Desde 2008, quando a instituição recebeu o status de universidade, houve um crescimento de 108,5\% no número de publicações. Nesse período, a instituição investiu na expansão tanto de cursos ofertados quanto de recursos humanos, o que pode explicar este fenômeno. Houve aumento de $180,0 \%$ no número de cursos de graduação, de alunos matriculados nos cursos de graduação $(90,1 \%)$, de alunos matriculados nos programas de pós-graduação stricto sensu (170,8\%), de mestres e doutores formados anualmente $(167,8 \%)$, de projetos de pesquisa $(182,0 \%)$, de grupos de pesquisa $(39,2 \%)$, de professores envolvidos com a pós-graduação $(71,1 \%)$ e de técnicos administrativos (113,0\%).

Com o objetivo de verificar se há relação entre o aumento da produção científica da UFCSPA e o aumento dos Grupos de Pesquisa, Docentes e Cursos de pós-graduação da universidade, procedeu-se a uma análise de correlação. $\mathrm{O}$ alto coeficiente de Pearson encontrado confirma a relação entre o aumento dos Grupos de Pesquisa CNPq e o aumento das publicações $\left(r^{2}=0,93530\right)$ bem como a relação entreos Docentes nos PPGs e as publicações $\left(r^{2}=0,94635\right)$. O aumento no número de cursos de pós-graduação, no entanto, apresentou correlação moderada $\left(r^{2}=0,70716\right)$. Estes dados indicam que os investimentos da instituição podem ter gerado resultados positivos quanto ao aumento de sua produção científica indexada na WoS.

O gráfico 2 apresenta a visualização do ritmo de crescimento da UFCSPA. Em relação a produção intelectual a universidade vem crescendo de forma exponencial, conforme o ajuste a linha com $R^{2}=0,94128$. Já os grupos de pesquisa, PPGs e docentes nos PPGs apresentaram um ajuste melhor a tendência polinomial de crescimento, com $R^{2}=0,8168, R^{2}=0,9596$ e $R^{2}=$ 0,88457 respectivamente. 
Maiara Bettio, Gonzalo Rubén Alvarez, Samile Andréa de Souza Vanz

Produção e colaboração científica da Universidade Federal de Ciências da Saúde de Porto Alegre

Gráfico 2 - Evolução temporal das publicações indexadas na WoS, Grupos de Pesquisa, PPGs e Docentes nos PPGs da UFCSPA (1979-2014)

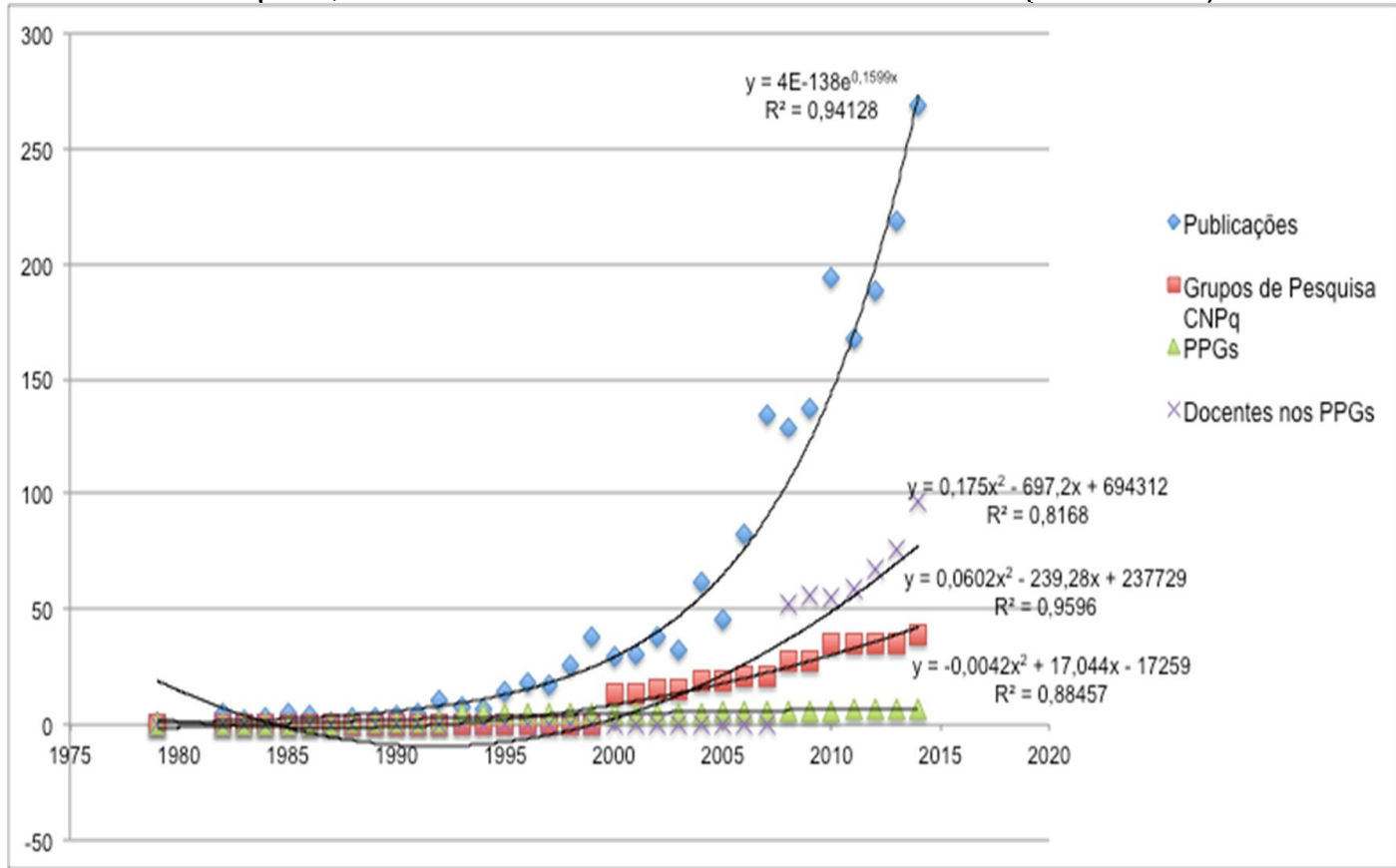

Fonte: dados da pesquisa

Quanto ao tipo de documento utilizado, o artigo é a modalidade de maior prestígio, representando 70,9\% das publicações indexadas. Em segundo lugar, está o resumo de evento, com $16,5 \%$ dos documentos, seguido pela carta $(5,1 \%)$ e pelo artigo de revisão (4,6\%). Tradicionalmente, o artigo de periódico é o canal de comunicação mais utilizado por pesquisadores em todo o mundo, sendo ainda mais evidente sua representatividade em ciência, tecnologia e medicina (MEADOWS, 1999). Os dados sugerem uma aproximação entre as características da produção científica da UFCSPA e os resultados obtidos por Saes (2005), que identificou que 69,0\% dos documentos das Ciências da Saúde brasileira foram publicados como artigos de periódico e 23,9\% como resumos em eventos.

Em relação ao idioma de publicação, uma expressiva maioria das publicações foi escrita em inglês, representando $91,3 \%$ dos documentos. Em segundo lugar está oportuguês (8,0\%), seguido dos idiomas espanhol $(0,7 \%)$ e francês $(0,1 \%)$. Há semelhanças nos índices observados por Vanz (2009) sobre a produção científica brasileira indexada na WoS entre 2004 e 2006, que indicam o uso do idioma inglês em $93,1 \%$ das publicações, enquanto o 
Maiara Bettio, Gonzalo Rubén Alvarez, Samile Andréa de Souza Vanz

Produção e colaboração científica da Universidade Federal de Ciências da Saúde de Porto Alegre

português alcançou 6,5\%. Brambilla (2011) também aponta a predominância do idioma inglês na análise da produção científica da UFRGS em Ciências da Saúde na WoS entre 2000 e 2009, cujos resultados alcançaram 93,7\% publicações em inglês e 5,8\% em português. Os indicadores de idioma observados manifestam uma forte internacionalização da pesquisa da UFCSPA, uma vez que mais de $90,0 \%$ das publicações foram divulgadas em inglês, considerada a língua oficial da comunicação científica (MEADOWS, 1999). O uso desse idioma, nesse sentido, possibilita que as publicações adquiram maior visibilidade, visto que os documentos são legíveis pela elite dos pesquisadores nacionais e internacionais (PACKER; MENEGHINI, 2006).

A análise dos periódicos identificou 694 canais utilizados para a publicação dos 1.932 documentos. Isso significa uma expressiva dispersão, indicativa de que os pesquisadores da UFCSPA transitam em um grande número de veículos de comunicação cientifica. Os 10 principais títulos de periódicos utilizados por autores da instituição entre 1979 e 2014 são apresentados a seguir.

Tabela 2 - Principais títulos de periódicos utilizados para publicação da produção científica da UFCSPA (1979-2014)

\begin{tabular}{|c|c|c|c|c|c|c|}
\hline & Título do periódico & $\begin{array}{l}\text { País de } \\
\text { origem }\end{array}$ & Idioma & FI 2014 & $\begin{array}{c}\text { N. de } \\
\text { documentos }\end{array}$ & $\%$ \\
\hline 1 & TransplantationProceedings & EUA & Inglês & 0,982 & 50 & 2,6 \\
\hline 2 & Arquivos de Neuro-Psiquiatria & Brasil & Inglês & 0,843 & 45 & 2,3 \\
\hline 3 & Anais Brasileiros de Dermatologia & Brasil & Português & 0,723 & 36 & 1,9 \\
\hline 4 & $\begin{array}{c}\text { Brazilian Journal of Medical and } \\
\text { Biological Research }\end{array}$ & Brasil & Inglês & 1,006 & 32 & 1,7 \\
\hline 5 & Jornal de Pediatria & Brasil & Inglês & 1,194 & 32 & 1,7 \\
\hline 6 & Jornal Brasileiro de Pneumologia & Brasil & Português & 1,017 & 31 & 1,6 \\
\hline 7 & Arquivos Brasileiros de Cardiologia & Brasil & Português & 1,021 & 26 & 1,3 \\
\hline 8 & LiverTransplantation & EUA & Inglês & 4,241 & 26 & 1,3 \\
\hline 9 & Hepatology & EUA & Inglês & 11,055 & 23 & 1,2 \\
\hline 10 & Hepato-Gastroenterology & Alemanha & Inglês & 0,928 & 22 & 1,1 \\
\hline
\end{tabular}

Fonte: dados da pesquisa

Inf. Inf., Londrina, v. 22, n. 1, p. 88 - 110, jan./abr., 2017. http:www.uel.br/revistas/informacao/ 
Destacam-se nas primeiras posições o Transplantation Proceedings e o Arquivos de Neuro-Psiquiatria, este último título indicado por Maia (2014) como o principal periódico utilizado para publicação dos artigos de autores brasileiros em Ciências da Saúde, indexados na base de dados Medline entre 1987 e 2011. A representatividade dos periódicos brasileiros merece destaque por reunir seis títulos entre os dez principais periódicos. Por outro lado, verificou-se que o Brasil é responsável por apenas $7,5 \%$ do total de periódicos que veiculam as publicações da instituição (52 títulos), enquanto que $92,5 \%$ dos periódicos são de origem estrangeira (642 títulos). Estes resultados demonstram o alcance internacional da produção científica da UFCSPA, tanto pela publicação em periódicos editados em outros países quanto pelo predomínio do idioma inglês.

A distribuição dos documentos por áreas de conhecimento foi analisadade acordo com a categorização e a terminologia utilizada pela WoS no campo WoS Categories para a classificação dos periódicos e, consequentemente, dos documentos por eles publicados. Foram identificadas 3.022 ocorrências de descritores, representando 110 categorias, o que indica uma dispersão dos assuntos de interesse dos pesquisadores da instituição, possivelmente associada à interdisciplinaridade das Ciências da Saúde.Os dados revelam que a Cirurgia é a área de maior produção, com 211 documentos, correspondente a 10,9\% da produção. Em seguida, destacam-se as áreas de Neurociências (8,9\%); Psiquiatria (6,9\%); Gastroenterologia\& Hepatologia (6,9\%); Pediatria $(6,2 \%)$ e Transplantes $(6,1 \%)$. Em um terceiro bloco, pode-se evidenciar as áreas de Sistema Cardíaco \& Cardiovascular (5,3\%); Imunologia (4,8\%); Farmacologia \& Farmácia (4,8\%); Sistema Respiratório (4,7\%); Urologia \& Nefrologia $(4,7 \%)$ e Endocrinologia \& Metabolismo(4,3\%). O Gráfico 3 apresenta o mapeamento da produção científica das áreas mais produtivas no período de 1979-2014, considerando o número de documentos publicados. 
Maiara Bettio, Gonzalo Rubén Alvarez, Samile Andréa de Souza Vanz

Produção e colaboração científica da Universidade Federal de Ciências da Saúde de Porto Alegre

Gráfico 3 - Áreas mais produtivas da UFCSPA e número de documentos publicados na WoS (1979-2014)

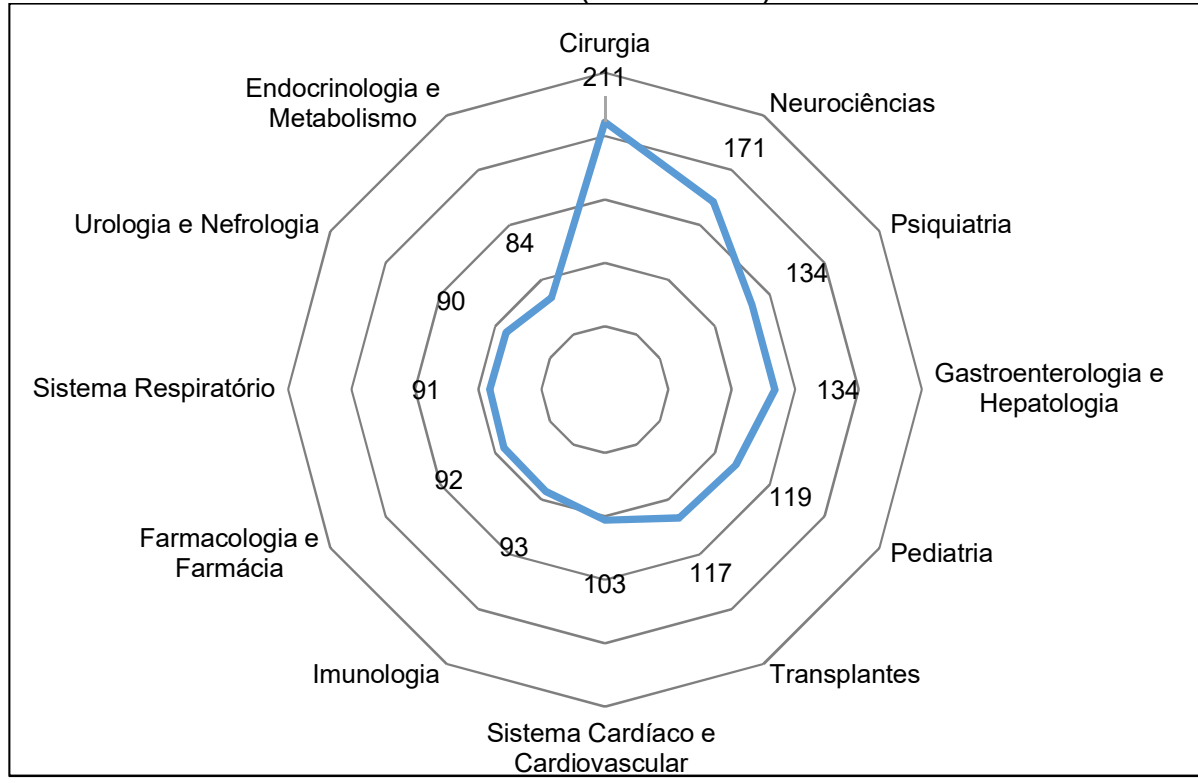

Fonte: dados da pesquisa

O destaque alcançado pela Cirurgia tem relação com a tradição médicocirúrgica no Brasil, apontada por Schwartzman (2001) como uma das áreas de conhecimento pioneiras entre as profissões estabelecidas desde o período colonial. Paralelamente, a história da UFCSPA tem como baseoensino médico em colaboração com a Santa Casa, onde muitos professores exercem atividades consagradas na área cirúrgica. Já a proeminência das Neurociências tem provável ligação com temas de interesse do programa de pós-graduação em Patologia, no qual as Neurociências constituem-se como uma das linhas de pesquisa, e do antigo programa de pós-graduação em Farmacologia, absorvido em 2002 pelo programa de pós-graduação em Ciências da Saúde. Os cursos de graduação em Psicologia e Medicina e osprogramas de residência médica em Neurologia também podem ter contribuído parao significativo número de publicaçõesalcançado pelas Neurociências, uma vez que dividem interesses comuns. Além disso, a área tem sido destacada por Santin, Brambilla e Stumpf (2013) como altamente produtiva e de forte expansão no cenário científico internacional. A área de Transplantes, por sua vez, se destaca entre os resultados pela proeminência alcançada pela Santa Casa, atualmente referência nacional no assunto. 
Finalizando os indicadores de produção científica, a análise de autoria revela que apenas $31,1 \%$ dos autores participaram da publicação de dois ou mais documentos. Foi bastante expressivo o númerode autores com apenas uma publicação, 68,9\%. Tais resultados indicam um alto índice de autores com baixo número de documentos publicados e podem estar associados ao fato de a UFCSPA ter um histórico recente de criação e desenvolvimento, bem como à sua incipiente tradição científica.

\subsection{Indicadores de Colaboração}

A análise dos indicadores de colaboração científica pretendeu identificar os padrões de coautoria estabelecidos nas atividades de pesquisa da UFCSPA, com prioridade à verificação dos níveis de colaboração e às relações entre países e instituições. No que se refere aos níveis de colaboração, verificou-se a predominância da coautoria na produção científica da universidade. Entre os 1.932 documentos analisados, apenas 1,4\% foram publicados em autoria única. A autoria múltipla, por sua vez, representa a maior proporção na amostra analisada, com $98,6 \%$ dos documentos publicados com dois ou mais autores. A média de autores por documento foi de 6,5 , índice aproximado ao identificado por Vanz (2009) acerca da produção científica nacional de 2004 a $2006(6,3)$ e superior à média mundial identificada por Glänzel e Schubert (2004) no ano $2000(4,6)$.

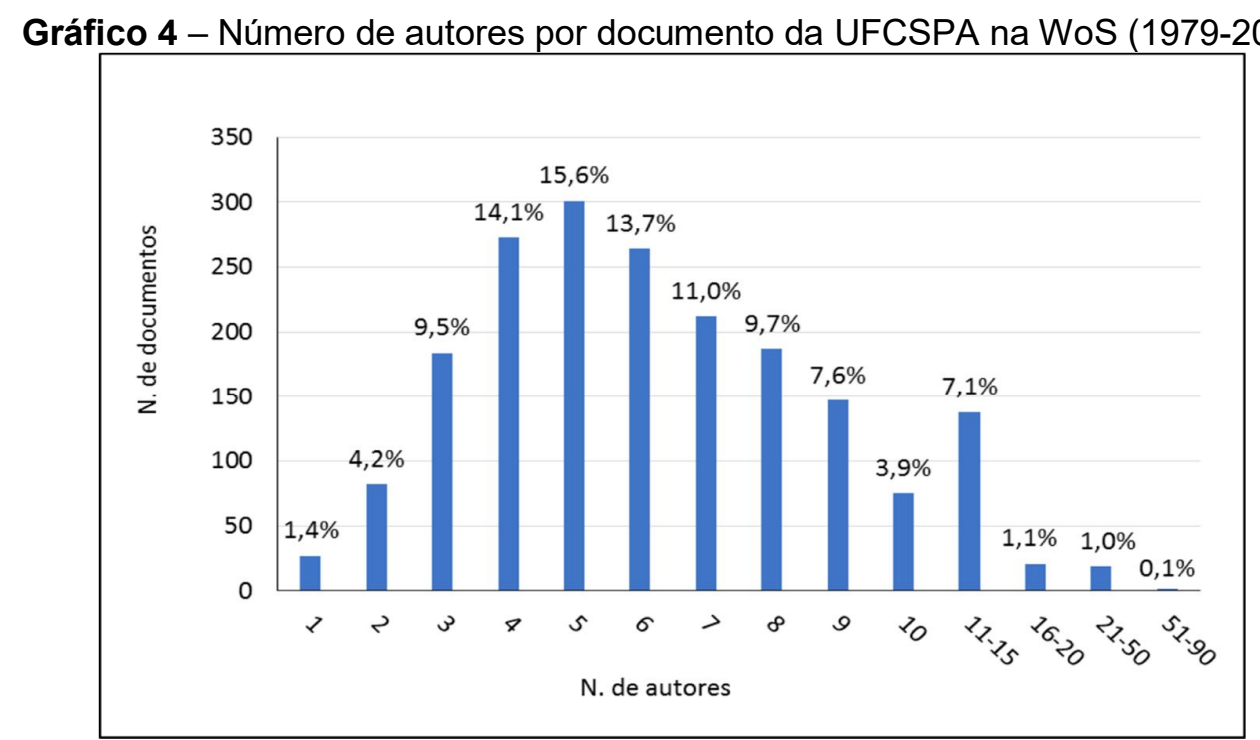

Fonte: dados da pesquisa 
Maiara Bettio, Gonzalo Rubén Alvarez, Samile Andréa de Souza Vanz

Produção e colaboração científica da Universidade Federal de Ciências da Saúde de Porto Alegre

O Gráfico 4 apresenta a distribuição dos documentos da UFCSPA na WoS pelo número de autores envolvidos. O nível de colaboração com maior incidência foi o de dois a dez autores por documento, com índice de $89,3 \%$, resultado que se assemelha ao encontrado por Brambilla (2011) sobre a produção científica das Ciências da Saúde da UFRGS entre 2000 a 2009, na qual $91,2 \%$ das publicações foram desenvolvidas neste mesmo nível de colaboração. Pode-se observar ainda, de forma mais específica,que os níveis de colaboração entre quatro, cinco e seis autores destacam-se como prática de publicação preferencial.

A prática de colaboração foi analisada também sob a perspectiva das relações da UFCSPA com outros países e instituições, no intuito de caracterizar as principais parcerias de pesquisa da universidade. Do total de 1.905 documentos produzidos em coautoria, 17,7\% contaram com a colaboração internacional. A Tabela 3 apresenta os 16 principais países identificados, a contagem das respectivas ocorrências e a porcentagem relativa ao total de documentos da pesquisae ao total de documentos em colaboração internacional.

Tabela 3 - Principais países colaboradores na produção científica da UFCSPA na WoS (1979-2014)

\begin{tabular}{|c|c|c|c|c|c|c|c|c|c|}
\hline & País & $\begin{array}{c}\text { N. de } \\
\text { documentos }\end{array}$ & $\%$ & $\% "$ & & País & $\begin{array}{c}\text { N. de } \\
\text { documentos }\end{array}$ & $\%$ & $\% "$ \\
\hline 1 & Estados Unidos & 182 & 9,4 & 53,7 & 9 & Alemanha & 14 & 0,7 & 4,1 \\
\hline 2 & Reino Unido & 65 & 3,4 & 19,2 & 10 & Austrália & 13 & 0,7 & 3,8 \\
\hline 3 & Canadá & 37 & 1,9 & 10,9 & 11 & México & 13 & 0,7 & 3,8 \\
\hline 4 & Espanha & 30 & 1,6 & 8,8 & 12 & Suiça & 12 & 0,6 & 3,5 \\
\hline 5 & França & 25 & 1,3 & 7,4 & 13 & Chile & 12 & 0,6 & 3,5 \\
\hline 6 & Argentina & 22 & 1,1 & 6,5 & 14 & Portugal & 12 & 0,6 & 3,5 \\
\hline 7 & Itália & 19 & 1,0 & 5,6 & 15 & Turquia & 10 & 0,5 & 2,9 \\
\hline 8 & Áustria & 15 & 0,8 & 4,4 & 16 & $\begin{array}{l}\text { África do } \\
\text { Sul }\end{array}$ & 10 & 0,5 & 2,9 \\
\hline
\end{tabular}

Fonte: dados da pesquisa

Nota: \%' se refere a porcentagem relativa ao total de documentos da pesquisa (1.932) e \%" se refere ao total de documentos em colaboração internacional (339)

Inf. Inf., Londrina, v. 22, n. 1, p. 88 - 110, jan./abr., 2017. http:www.uel.br/revistas/informacao/ 
Os resultados mostram que os Estados Unidos se constituem como o principal país coautor nos documentos publicados pela UFCSPA $(9,4 \%)$. Esse índice eleva-se a $53,8 \%$ quando o número de documentos do país é considerado em relação ao número de documentos produzidos em colaboração internacional (339). A segunda colocação é ocupada pelo Reino Unido (3,4\%), com destaque ainda para Canadá (1,9\%), Espanha (1,6\%), França $(1,3 \%)$ e Argentina $(1,1 \%)$. As práticas de colaboração científica na UFCSPA seguem a tendência da produção científica brasileira indicada por Vanz (2009), segundo a qual os Estados Unidos são apontados como o mais importante colaborador em pesquisas do Brasil.

O índice de $17,7 \%$ de documentos produzidos pela UFCSPA em colaboração internacional revela-se similar ao identificado na produção cientifica mundial. De acordo com Glänzel e Schubert (2004), esse era o percentual de artigos publicados por dois ou mais países indexados no Science Citation Index da WoS no ano 2000. No entanto, se comparado aos resultados obtidos pela produção científica brasileira, o índice mostra-se inferior. Meis e Leta (1996) apontam que nas publicações de 1991 a 1993 a colaboração científica brasileira com outros países atingiu $26,6 \%$, enquanto Vanz (2009), no período 2004-2006, mostra que o índice chegou a 30,3\%.

Outro aspecto analisado diz respeito às instituições coautoras. Dos 1.905 documentos produzidos em colaboração, $86,8 \%$ contavam com duas ou mais instituições, enquanto que em $13,2 \%$ dos documentos apenas uma instituição foi indicada. Ao total, identificou-se 982 instituições, revelando a diversidade institucional presente nas publicações em coautoria. A Tabela 4 lista as 25 principais instituições parceiras e o correspondente Estado, juntamente com o número de documentos publicados em colaboração e a respectiva porcentagem em relação ao total de documentos deste estudo. 
Maiara Bettio, Gonzalo Rubén Alvarez, Samile Andréa de Souza Vanz

Produção e colaboração científica da Universidade Federal de Ciências da Saúde de

Porto Alegre

Tabela 4 - Principais instituições colaboradoras na produção científica da UFCSPA na WoS (1979-2014)

\begin{tabular}{|c|c|c|c|c|}
\hline & Instituição & Estado & N. de documentos & $\%$ \\
\hline 1 & Universidade Federal do Rio Grande do Sul (UFRGS) & RS & 804 & 41,6 \\
\hline 2 & Santa Casa de Misericórdia de Porto Alegre & RS & 598 & 31,0 \\
\hline 3 & Universidade de São Paulo (USP) & SP & 148 & 7,7 \\
\hline 4 & Pontifícia Universidade Católica do Rio Grande do Sul (PUCRS) & RS & 113 & 5,8 \\
\hline 5 & Instituto de Cardiologia do Rio Grande do Sul & RS & 100 & 5,2 \\
\hline 6 & Universidade Federal de São Paulo (UNIFESP) & SP & 96 & 5,0 \\
\hline 7 & Universidade Luterana do Brasil (ULBRA) & RS & 95 & 4,9 \\
\hline 8 & Universidade Federal do Rio de Janeiro (UFRJ) & RJ & 89 & 4,6 \\
\hline 9 & Grupo Hospitalar Conceição & RS & 61 & 3,2 \\
\hline 10 & Universidade Federal de Pelotas (UFPEL) & RS & 46 & 2,4 \\
\hline 11 & Universidade de Caxias do Sul (UCS) & RS & 38 & 2,0 \\
\hline 12 & Universidade FEEVALE (FEEVALE) & RS & 36 & 1,9 \\
\hline 13 & Universidade Federal de Santa Catarina (UFSC) & SC & 35 & 1,8 \\
\hline 14 & Hospital Mãe de Deus & RS & 34 & 1,8 \\
\hline 15 & Hospital Moinhos de Vento & RS & 33 & 1,7 \\
\hline 16 & Universidade Federal de Santa Maria (UFSM) & RS & 33 & 1,7 \\
\hline 17 & Centro Universitário Metodista (IPA) & RS & 30 & 1,6 \\
\hline 18 & Universidade Federal do Paraná (UFPR) & PR & 29 & 1,5 \\
\hline 29 & Harvard University & - & 27 & 1,4 \\
\hline 20 & Universidade do Vale do Rio dos Sinos (UNISINOS) & RS & 25 & 1,3 \\
\hline 21 & Universidade Federal de Minas Gerais (UFMG) & MG & 24 & 1,2 \\
\hline 22 & Hospital Materno Infantil Presidente Vargas (HMIPV) & RS & 22 & 1,1 \\
\hline 23 & Liverpool Heart \&Chest Hospital & -- & 21 & 1,1 \\
\hline 24 & Universidade Católica de Pelotas (UCPEL) & RS & 21 & 1,1 \\
\hline 25 & Fundação Universidade Federal de Rio Grande (FURG) & $\mathrm{RS}$ & 20 & 1,0 \\
\hline
\end{tabular}

Fonte: dados da pesquisa

Observa-se entre as instituições coautoras a presença expressiva de universidades nacionais, especialmente as públicas, além de algumas instituições hospitalares, indo de encontro aos resultados observados nas Ciências da Saúde no Brasil por Maia (2014). A UFRGS se destaca como a instituição com maior número de documentos publicados em colaboração com a UFCSPA (41,6\%), seguida pela Santa Casa de Misericórdia de Porto Alegre $(31,0 \%)$. Merece destaque ainda a Universidade de São Paulo (USP) $(7,7 \%)$, a

Inf. Inf., Londrina, v. 22, n. 1, p. 88 - 110, jan./abr., 2017. http:www.uel.br/revistas/informacao/ 
Pontifícia Universidade Católica do Rio Grande do Sul (PUCRS) (5,8\%), o Instituto de Cardiologia do Rio Grande do Sul $(5,2 \%)$ e a Universidade Federal de São Paulo (UNIFESP) (5,0\%). Estes resultados mostram que mais de dois terços da produção científica da UFCSPA indexada na WoS $(67,4 \%)$ foi publicada em colaboração com duas proeminentes instituições em suas áreas de atuação (ensino universitário/serviço hospitalar). A UFRGS é apontada como líder em produção científica em Ciências da Saúde no estado (MAIA, 2014), enquanto que a Santa Casa constitui uma instituição de referência nacional em Saúde. Além disso, ambas estão localizadas na cidade de Porto Alegre, revelando uma forte regionalização nas práticas de colaboração da UFCSPA. Tal aspecto fica ainda mais evidente ao se considerar que um grande número de instituições colaboradoras estão no estado do Rio Grande do Sul, contando com cursos de graduação ou de pós-graduação em Ciências da Saúdee com vínculos formais de colaboração firmados com a UFCSPA. A importância da proximidade geográfica na produção do conhecimento e nas relações de colaboração estabelecidas entre os pesquisadores já foi apresentada por Sidone, Haddad e Mena-Chalco (2016) e é um aspecto que merece ser acompanhado em futuros estudos acerca da produção da UFCSPA.

\section{CONSIDERAÇÕES FINAIS}

A análise dos 1.932 registros identificados possibilitou a caracterizaçãoda produção científica da UFCSPA na Web of Science entre 1979 e 2014 de acordo com indicadores bibliométricos de produção e de colaboração. Apesar do desenvolvimento tardio, o número de publicações da instituição apresentou crescimento anual significativo no período analisado, de $268,0 \%$ no total, com taxa média de $33,5 \%$ ao ano e ajustado ao modelo exponencial. Destacou-se o período compreendido entre 2008 e 2014, coincidente à época de atribuição do status de universidade à instituição, e no qual foram publicados mais de $60,0 \%$ de todos os documentos analisados. A análise de correlação confirma a relação entre o aumento dos Grupos de Pesquisa CNPq e o aumento das publicações, bem como a relação entre os docentes nos PPGs e as publicações, indicando que os investimentos da 
instituição podem ter gerado resultados positivos quanto ao aumento de sua produção científica indexada na WoS.

$\mathrm{O}$ artigo de periódico constituiu a tipologia de documento de maior prestígio entre os pesquisadores da UFCSPA, utilizado em $70,9 \%$ das publicações. Em consonância com estudos sobre a produção científica brasileira em Ciências da Saúde, os documentos da UFCSPA foram publicados, em sua expressiva maioria $(91,3 \%)$, no idioma inglês. Tais resultados indicam uma forte internacionalização e visibilidade das pesquisas produzidas na instituição.

Verificou-se a presença de 694 periódicos utilizados, apontando uma expressiva dispersão indicativa de que os pesquisadores da UFCSPA transitam entre um grande número de veículos de comunicação científica. Além disso, $92,5 \%$ dos periódicos são de origem estrangeira e apenas $7,5 \%$ são periódicos nacionais. Por outro lado, na análise dos dezcom maior número de documentos da UFCSPA, houve uma presença significativa de periódicos brasileiros.

As áreas de conhecimento com maior produtividade foram a Cirurgia $(10,9 \%)$ e as Neurociências $(8,9 \%)$, seguidas da Psiquiatria (6,9\%), da Gastroenterologia \& Hepatologia $(6,9 \%)$, da Pediatria $(6,2 \%)$ e da área de Transplantes $(6,1 \%)$. A proeminência da primeira se fundamenta na relação histórica e institucional da UFCSPA com a Santa Casa de Misericórdia de Porto Alegre, bem como à tradição do ensino médico na universidade. As Neurociências, por sua vez, se destacam por sua vinculação aos principais programas de pós-graduação em atividade na instituição.

Constatou-se que $68,9 \%$ dos autores publicaram apenas um documento e $31,1 \%$ dos autores publicaram dois ou mais documentos, indicando um alto índice de autores com baixo número de documentos publicados,que pode estar associado ao fato de a UFCSPA ter um histórico recente de criação e desenvolvimento, bem como à sua incipiente tradição científica.

Quanto aos níveis de colaboração, a autoria múltipla predomina entre os resultados, representando $98,6 \%$ dos documentos. Destes, $82,3 \%$ foram produzidos em coautoria com autores brasileiros e $17,7 \%$ em coautoria com autores de outros países, sugerindo que as práticas de colaboração internacional da UFCSPA estão de acordo àquelas identificadas na produção 
científica mundial, mas em patamar inferior às observadas na produção científica brasileira. Os Estados Unidos se constituem como o principal país coautor nos documentos publicados, com $9,4 \%$, índice que passa a $53,7 \%$ quando o número de documentos do país é considerado em relação ao número de documentos produzidos em colaboração internacional.

Dos 1.905 documentos produzidos em colaboração, 86,8\% contavam com duas ou mais instituições coautoras. Ao total, identificou-se 982 instituições, sendo que entre as principaispredominam instituições universitárias nacionais, especialmente as públicas, além de algumas instituições hospitalares. A UFRGS foi a instituição com maior número de documentos publicados em colaboração com a UFCSPA, seguida pela Santa Casa de Misericórdia de Porto Alegre. Isso mostra que mais de dois terços da produção científica foi publicada em colaboração com duas proeminentes instituições localizadas em Porto Alegre, revelando uma forte regionalização nas práticas de colaboração científica da universidade.

O estudo evidenciou um significativo crescimento no número de publicações da UFCSPA durante o período analisado, assim como a diversidade de autores, periódicos, países e instituições envolvidas em suas práticas de pesquisa. Embora restrito à análise da produção e da colaboração científica representadana WoS, acredita-se que o estudo contribui para o conhecimento de importantes dimensões das pesquisas realizadas pela universidade, assim como parao aperfeiçoamento de suas políticas de desenvolvimento e de divulgação científicas.

Além disso, a baixa recuperação de registros na WoS entre 1961-1978 apresenta-se como uma limitação que merece ser objeto de análise futura, inclinada especialmente à busca de registros através do campo "Author". Assim sendo, consideram-se válidos estudos futuros que possam aprofundar e ampliar os resultados apresentados, especialmente no que tange a redes sociais estabelecidas entre pesquisadores e a indicadores do impacto da produção científica da UFCSPA. 
Maiara Bettio, Gonzalo Rubén Alvarez, Samile Andréa de Souza Vanz

Produção e colaboração científica da Universidade Federal de Ciências da Saúde de Porto Alegre

\section{REFERÊNCIAS}

ALMEIDA, E. C. E. de; GUIMARÃES, J. A. Brazil's growing production of scientific articles: how are we doing with review articles and other qualitative indicators?

Scientometrics, Amsterdan, v. 97, n. 2, p. 287-315, nov. 2013.

BONAMIGO, T. P.; OLIVEIRA, M. da C. (Org.). Memórias da criação da Fundação Faculdade Federal de Ciências Médicas de Porto Alegre. Porto Alegre: [s.n.], 2007.

BRAMBILLA, S. D. S. Produção Científica da UFRGS representada na WOS (20002009). 2011. 216 f. Tese (Doutorado em Comunicação e Informação) - Faculdade de Biblioteconomia e Comunicação, Universidade Federal do Rio Grande do Sul, Porto Alegre, 2011.

CNPq - CONSELHO NACIONAL DE DESENVOLVIMENTO CIENTÍFICO E TECNOLÓGICO. Diretório dos grupos de pesquisa no Brasil. [2015]. Disponível em: <http://lattes.cnpq.br/web/dgp/painel-dgp/>. Acesso em: 17 jun. 2015.

FAPESP - FUNDAÇÃO DE AMPARO À PESQUISA DO ESTADO DE SÃO PAULO. Análise da produção científica a partir de publicações em periódicos especializados. In:_ Indicadores de ciência, tecnologia e inovação em São Paulo 2010. São Paulo: FAPESP, v.1, 2011. cap. 4.

GLÄNZEL, W.; SCHUBERT, A. Analyzing scientific networks through co authorship. In: MOED, H. F.; GLÄNZEL, W.; SCHMOCH, U. Hand book of Quantitative Science and Technology Research. Netherlands: Kluwer Academic, 2004. p. 257-276.

MACIAS-CHAPULA, C. A. O papel da informetria e da cienciometria e sua perspectiva nacional e internacional. Ciência da Informação, Brasília, v. 27, n. 2, p. 134-140, maio/ago. 1998.

MAIA, M. de F. S. Comunicação científica em ciências da saúde no Brasil: estrutura e dinâmica da produção e indícios de vitalidade. 2014. $211 \mathrm{f}$. Tese (Doutorado em Comunicação e Informação) - Faculdade de Biblioteconomia e Comunicação, Universidade Federal do Rio Grande do Sul, Porto Alegre, 2014.

MEADOWS, A. J. A comunicação científica. Brasília: Briquet de Lemos Livros, 1999.

MEIS, L. de; LETA, J. O perfil da ciência brasileira. Rio de Janeiro: Editora UFRJ, 1996.

PACKER, A. L.; MENEGHINI, R. Visibilidade da produção científica. In: POBLACIÓN, D. A.; WITTER, G. P.; SILVA, J. F. M. Comunicação e produção científica: contexto, indicadores e avaliação. São Paulo: Angellara, 2006. p. 235-259.

SAES, S. G. Aplicação de métodos bibliométricos e da "co-wordanalysis" na avaliação da literatura científica brasileira em ciências da saúde de 1990 a 2002. 2005. 183 f. Tese (Doutorado em Saúde Pública) - Faculdade de Saúde Pública, Universidade de São Paulo, São Paulo, 2005.

SANTIN, D. M.; BRAMBILLA, S. D. S.; STUMPF, I. R. C. Produção científica em Neurociências da UFRGS indexada na Web of Science: 2000-2009. Liinc em Revista, Rio de Janeiro, v. 9, n. 1, p. 66-84, maio 2013. 
Maiara Bettio, Gonzalo Rubén Alvarez, Samile Andréa de Souza Vanz

Produção e colaboração científica da Universidade Federal de Ciências da Saúde de Porto Alegre

SCHWARTZMAN, S. Um espaço para a ciência: a formação da comunidade científica no Brasil. Brasília: Ministério da Ciência e Tecnologia, 2001.

SIDONE, O. J. G.; HADDAD, E. A.; MENA-CHALCO, J. P. Scholarly publication and collaboration in Brazil: the role of geography. Journal of the Association for Information Science and Technology, 2016.

STUMPF, I. R. C. A comunicação da ciência na universidade: o caso da UFRGS. In: MUELLER, S. P. M.; PASSOS, E. J. L. (Org.). Comunicação científica. Brasília, DF: Depart. de Ciência da Informação Univers. de Brasília, 2000. p. 107-121.

UFCSPA - UNIVERSIDADE FEDERAL DE CIÊNCIAS DA SAÚDE DE PORTO

ALEGRE. Relatório de gestão do exercício de 2013. 2014. Disponível em:

<http://www.ufcspa.edu.br/ufcspa/administracao/planodegestao/relatorio-de-gestao2013.pdf>. Acesso em: 05 maio 2016.

Histórico. [2015a]. Disponível

em:<http://www.ufcspa.edu.br/index.php/historico>. Acesso em: 28 abr. 2016.

Relatório de gestão do exercício de 2014. 2015b. Disponível em:

$\overline{<h t t p: / / w w w . u f c s p a . e d u . b r / u f c s p a / a d m i n i s t r a c a o / p l a n o d e g e s t a o / r e l a t o r i o-d e-g e s t a o-~}$ 2014.pdf>. Acesso em: 14 ago. 2016.

UFCSPA inaugura novo prédio no campus. 2015c. Disponível em: <http://www.ufcspa.edu.br/index.php/sala-de-pautas/3752-ufcspa-inaugura-novopredio-no-campus>. Acesso em: 1 jun. 2016.

Plano de metas das unidades. 2015d. Disponível em:

<http://www.ufcspa.edu.br/ufcspa/administracao/planodegestao/plano-de-metas-dasunidades.pdf $>$. Acesso em: 05 maio 2016.

VANZ, S. A. de S. As redes de colaboração científica no Brasil. 2009. 204 f.Tese (Doutorado em Comunicação e Informação) - Faculdade de Biblioteconomia e Comunicação, Universidade Federal do Rio Grande do Sul, Porto Alegre, 2009.

VELHO, L. A. Avaliação do desempenho cientifico. Cadernos USP, São Paulo, n. 1, p. 22-40, 1986.

\section{Title}

Scientific output and collaboration of Universidade Federal de Ciências da Saúde de Porto Alegre

\section{Abstract:}

Introduction: Mapping the scientific output of educational and research institutions is important for policy planning and performance evaluation. Objective: To identify and characterize the scientific output of Universidade Federal de Ciências da Saúde de Porto Alegre (UFCSPA) through bibliometric indicators of production and collaboration. Methodology: Bibliometric analysis of 1932 documents indexed on the Web of Science (WoS) database from 1961to 2014 using Bib excel and Excel 2010. Results: The analysis reveals a meaningful annual growth (33.5\% by year), highlighting the 
Maiara Bettio, Gonzalo Rubén Alvarez, Samile Andréa de Souza Vanz

Produção e colaboração científica da Universidade Federal de Ciências da Saúde de

Porto Alegre

period from 2008 to 2014 . $70.9 \%$ of the publications are articles and $91.3 \%$ of the documents were published in English. The most productive fields of knowledge were Surgery and Neurosciences. Multiple co authorship has a clearly predominance $(98.6 \%)$ and $17.7 \%$ of this amount are in international collaboration type. The United States of America, United Kingdom, Canada, Spain, France, and Argentina are the main partners. Among the 1905 documents in coauthor ship collaboration, $86.8 \%$ have two or more institutions involved with a predominance of national public universities followed by hospitals. Universidade Federal do Rio Grande do Sul (UFRGS) (41.6\%) and Santa Casa de Misericórdia de Porto Alegre (31.0\%) are the main partners. Conclusions: It concludes that the increase of research groups and researchers contributes to UFCSPA scientific output growth.

Keywords: Bibliometrics. Scientific productivity. Scientific collaboration. Universidade Federal de Ciências da Saúde de Porto Alegre. Medicine.

\section{Titulo}

Producción y colaboración científica de la Universidade Federal de Ciências da Saúde de Porto Alegre

\section{Resumen:}

Introducción: El mapeo de la producción científica de las instituciones de enseñanza e investigación es fundamental para planeamiento de políticas y evaluación de desempeño. Objetivo: Identificar y caracterizar la producción científica de la Universidade Federal de Ciências da Saúde de Porto Alegre (UFCSPA) a través de indicadores bibliométricos de producción y colaboración. Metodología: Análisis bibliométrico de 1.932 documentos indexados en la base de datos Web of Science (WoS) de 1961 a 2014, con ayuda de los software Bibexcel y Excel 2010. Resultados: El análisis muestra el crecimiento anual significativo de las publicaciones de la institución (33,5\% al año), destacándose el período 2008-2014. El artículo fue utilizado en $70,9 \%$ de las publicaciones y $91,3 \%$ de los documentos fueron publicados en el idioma inglés. Las áreas de conocimiento con mayor productividad fueron la Cirugía y las Neurociencias. La autoría múltiple se muestra predominante (98,6\%). De éstos, $17,7 \%$ se presentan en coautoría internacional. Estados Unidos, Reino Unido, Canadá, España, Francia y Argentina son los principales socios. De los 1.905 documentos con colaboración, $86,8 \%$ tienen dos o más instituciones coautoras, con predominio de las universidades nacionales públicas, además de las instituciones hospitalarias. UFRGS $(41,6 \%)$ y Santa Casa de Misericórdia de Porto Alegre $(31,0 \%)$ se destacan como las principales socias. Conclusiones: Concluye que el aumento de los grupos de investigacióny de los docentes investigadores contribuyó para el crecimiento de la producción intelectual de la UFCSPA.

Palabras-clave: Bibliometría. Productividad científica. Colaboración científica. Universidade Federal de Ciências da Saúde de Porto Alegre. Medicina.

Recebido: 12.02 .2016

Aceito: 25.03.2017 